\title{
The Distribution of Sister Chromatid Exchanges and Chromosomal Aberrations Induced by 5-Fluorodeoxyuridine and Ethyl Methanesulfonate in the Euchromatin and Heterochromatin of Chinese Hamster Cells
}

\author{
Akira Sono and Kengo Sakaguchi* \\ Research Laboratories, Toyo Jozo Co., Ltd., Ohito-cho, Tagata-gun, Shizuoka- \\ ken, Japan. and* Department of Biology, University of California at San Diego, \\ La Jolla, California 92093, U.S.A.
}

\begin{abstract}
The frequencies and distributions of sister chromatid exchanges and chromosomal aberrations induced by 5-fluorodeoxyuridine and ethyl methanesulfonate on cultured Chinese hamster cells were investigated.

The distributions of sister chromatid exchanges and chromosomal aberrations induced by both agents in the early $\mathrm{S}$ phase were entirely different. Sister chromatid exchanges were distributed uniformly at a relatively higher incidence in the euchromatic regions, whereas chromosomal aberrations were more frequent in heterochromatin.

Combined treatment with 5-fluorodeoxyuridine and ethyl methanesulfonate significantly enhanced the frequency of sister chromatid exchanges without changing their distribution along the length of the chromosome.
\end{abstract}

Our previous studies on the relationship between SCEs and chromosomal aberrations $(13,14)$ showed that exposure of cultured mammalian cells to various nucleic acid synthesis inhibitors increases the frequencies of SCEs and aberrations to some extent, depending on the stage of cell cycle, with different degrees of fluctuation in the dose-response curves of both events, and that there were few chromosomal breaks and gaps that share breakpoints with SCEs.

Several workers $(1,2,6,10,16,21)$ have reported (on the basis of analysis of spontaneous SCEs in certain established cell lines derived from normal mammalian cells) that relatively few SCEs occur in C-band regions, although the frequency is rather high at junctions between the C-band positive and negative regions. In Bloom's syndrome, cells with an unusually high frequency of spontaneous SCEs and chromosomal breakages, do not necessarily show breakpoints that coincide (12). Carrano and Johnston (3), however, reported that in mitomycin C-treated Indian muntjac cells the SCEs in the euchromatic regions were proportional to the DNA content, and that SCEs at junctions between the heterochromatic neck and the euchromatic arms of the $\mathrm{X}$ chromosome occurred more frequently than expected.

This present paper deals with SCEs and chromosomal aberrations in Chinese hamster cells exposed to FUdR and EMS, alone or in combination. Special attention

\footnotetext{
Abbreviations used; SCE, sister chromatid exchange; BUdR, 5-bromodeoxyuridine; FUdR, 5-fluorodeoxyuridine; EMS, ethyl methanesulfonate.
} 
has been paid to the frequencies and distributions of both events in the heterochromatic and euchromatic chromosomal regions, when cells were treated at different phases of DNA synthesis.

\section{MATERIALS AND METHODS}

Cell Culture. A Chinese hamster cell line, DON D-6 (the gift of Dr. Kato), was used. This cell line had 22 chromosomes with a pseudodiploid karyotype, as reported previously by Kato and Yosida (7). The cells were cultured at $37^{\circ} \mathrm{C}$ in an atmosphere of 5 per cent $\mathrm{CO}_{2}$ in Eagle's minimal essential medium (Nissui Seiyaku Co., Tokyo) supplemented with $10 \%$ fetal calf serum (Grand Island Biological Co., N. Y., U. S. A.) heat-inactivated at $56^{\circ} \mathrm{C}$ for $30 \mathrm{~min}, 1 \mathrm{mM}$ sodium pyruvate, $0.2 \mathrm{mM}$ serine, $100 \mathrm{U} / \mathrm{ml}$ penicillin and $100 \mu \mathrm{g} / \mathrm{ml}$ streptomycin.

BUdR-labeling and Chromosomal preparation. To detect SCEs, we incubated exponentially growing cells in medium containing $10^{-5} \mathrm{M}$ BUdR (BUdR-medium) for two cell cycles. The duration of the cell cycle and the population doubling time under these culture conditions have been reported in a previous paper (15). BUdR, FUdR and EMS were purchased from Sigma Chemical Co., St. Louis, Mo., U. S. A. All reagents were dissolved, at appropriate concentrations, in the medium immediately before use, then added to the culture for the $3 \mathrm{~h}$ of the second S-phase, or for the entire second replication cycle, in the BUdR-medium (see legends to Tables 1 and 2). Colcemid (GIBCo, $0.05 \mu \mathrm{g} / \mathrm{ml}$ ) was added to the culture $2 \mathrm{~h}$ before collection. Cells were collected by trypsinization and treated with hypotonic $\mathrm{KCl}$ $(0.075 \mathrm{M})$ for $10 \mathrm{~min}$. Chromosomal preparations were made according to the standard air-drying technique. The fluorescent plus Giemsa method (19) was used for the differential staining of sister chromatids.

Measurement of C-band heterochromatin and SCE analysis. The proportion of C-band heterochromatin, determined by the barium hydroxide-saline-Giemsa method (18), was estimated by measuring the length and distance of the C-bands from the centromere as the proportion of total chromosome length on photographs of 25 metaphases. The location of SCEs and chromosomal aberrations on each chromosome (Fig. 1) was measured on randomly selected photographs of 25 complete and well spread second division metaphases. SCEs and break point were plotted on the idiogram at $0.5 \%$ intervals of the total length of each chromosome. Chromosomes 10 and 11 were too small for exact analysis and were omitted from this examination. Gaps at the secondary constriction of $\mathrm{X}$ chromosome were not regarded as aberrations.

\section{RESULTS}

The karyotype and C-banding pattern of this cell line are shown in Fig. 1. Each arm of chromosomes 1 and 2 had an interstitial or terminal C-band. The X chromosome had a secondary constriction at the distal portion of the totally heterochromatic long arm. The Y chromosome and chromosomes 10 and 11 were totally C-band positive. Chromosomes 5, 7 and 8, and two marker chromosomes, $\mathbf{M}_{1}$ and $\mathbf{M}_{2}$ (which were assumed to be formed by centric fusion of two acrocentric chromosomes 6 or a translocation involving a chromosome 6), had prominent centromeric heterochromatin.

Table 1 shows the effects of FUdR and EMS, alone or in combination, on the frequency of SCEs and chromosomal aberrations. EMS was confirmed to be a strong 


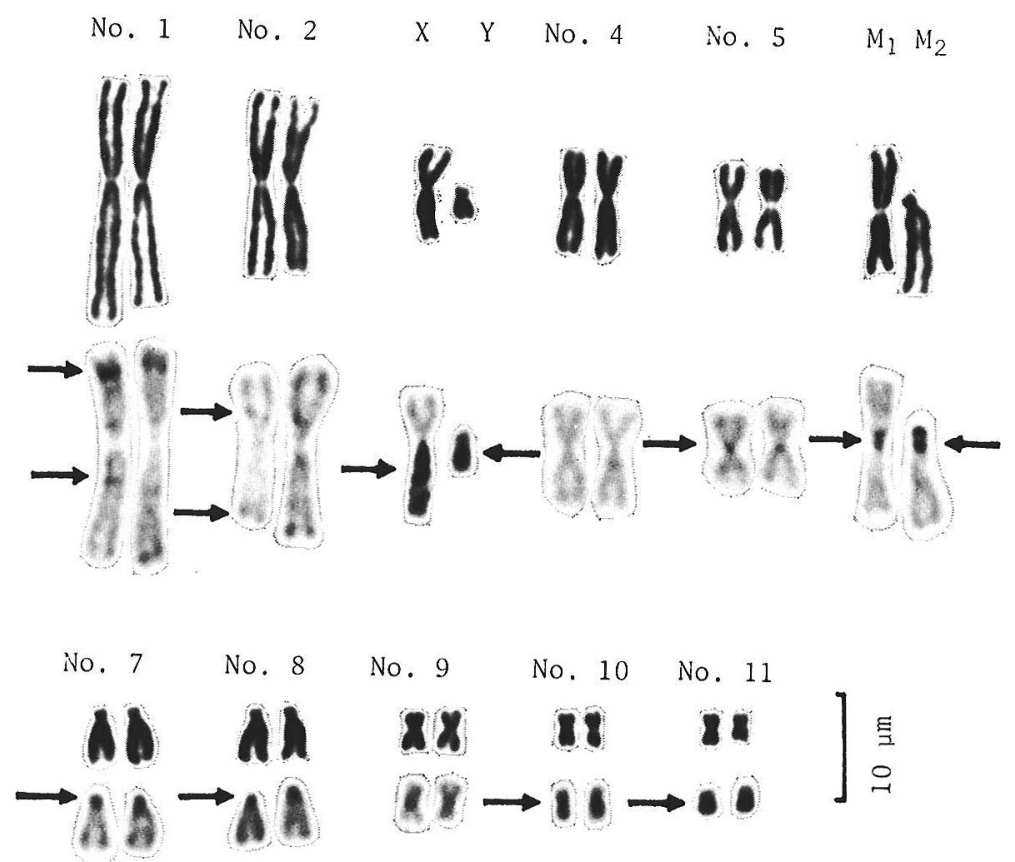

Fig. 1. A C-banded karyotype of the D-6 cell arranged by the classification system given in ref. 6 . Arrows indicate the C-band regions.

inducer of SCEs and chromosomal aberrations, and the lesions caused by this agent that lead to SCEs seem to be long-lived (Table 1, and ref. 14). FUdR also induced both SCEs and chromosomal aberrations as reported previously (14). Simultaneous treatment with $10^{-5} \mathrm{M}$ FUdR and $10^{-3} \mathrm{M}$ EMS markedly increased the SCE frequen-

TABLE 1. EFFECTS OF FUDR AND EMS ON THE INDUCTION OF SCES AND CHROMOSOMAL ABERRATION

\begin{tabular}{|c|c|c|c|c|}
\hline \multirow{2}{*}{ Treatment } & \multirow{2}{*}[\mathrm{M}]{} & \multirow{2}{*}{$\begin{array}{l}\text { Chromosomal abberations } \\
\text { per cell }\end{array}$} & \multicolumn{2}{|c|}{ SCEs per cell (Mean \pm S.D.) } \\
\hline & & & Observed & Expected \\
\hline Control (BUdR & $\left.10^{-5}\right)$ & 0.05 & $4.73 \pm 1.12$ & - \\
\hline \multirow[t]{2}{*}{ EMS } & $10^{-3}$ & 0.52 & $22.92 \pm 4.46(18.19)^{b}$ & - \\
\hline & $10^{-4}$ & 0.22 & $8.48 \pm 1.76(3.75)$ & - \\
\hline \multirow[t]{2}{*}{ FUdR } & $10^{-5}$ & 0.26 & $7.48 \pm 1.75(2.75)$ & - \\
\hline & $10^{-6}$ & 0.22 & $6.22 \pm 1.44(1.49)$ & - \\
\hline \multicolumn{2}{|c|}{ EMS $10^{-3}+$ FUdR $10^{-5}$} & 0.68 & $31.95 \pm 3.39^{a}$ & $25.67^{c}$ \\
\hline "I & $10^{-6}$ & 0.63 & $23.08 \pm 2.62$ & 24.41 \\
\hline \multicolumn{2}{|c|}{ EMS $10^{-4}+$ FUdR $10^{-5}$} & 0.55 & $11.96 \pm 2.22$ & 11.23 \\
\hline "I & $10^{-6}$ & 0.59 & $8.83 \pm 1.87$ & 9.97 \\
\hline
\end{tabular}

Cells cultured for $15 \mathrm{~h}$ in medium containing $10^{-5} \mathrm{M}$ BUdR were treated with FUdR and EMS alone, or in combination, for the second replication cycle in the presence of BUdR.

a Significant increase from the expected value $(\mathrm{p} \leq 0.05)$.

$b$ Each value shown in parentheses is the induced SCE frequency after subtracting the background value, 4.73 .

c The sum of the induced and spontaneous (background) SCE frequencies was taken as the expected value. 


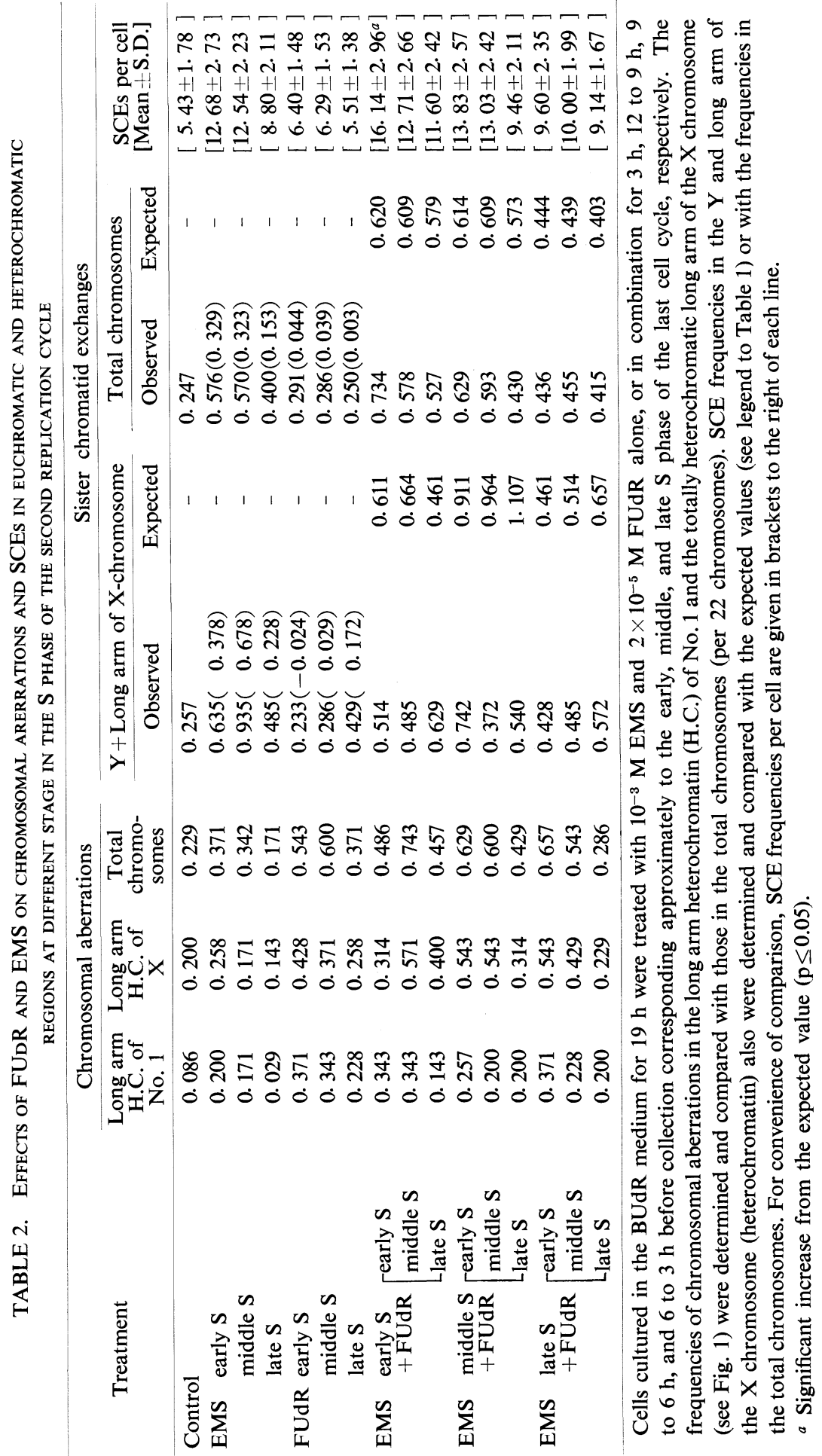




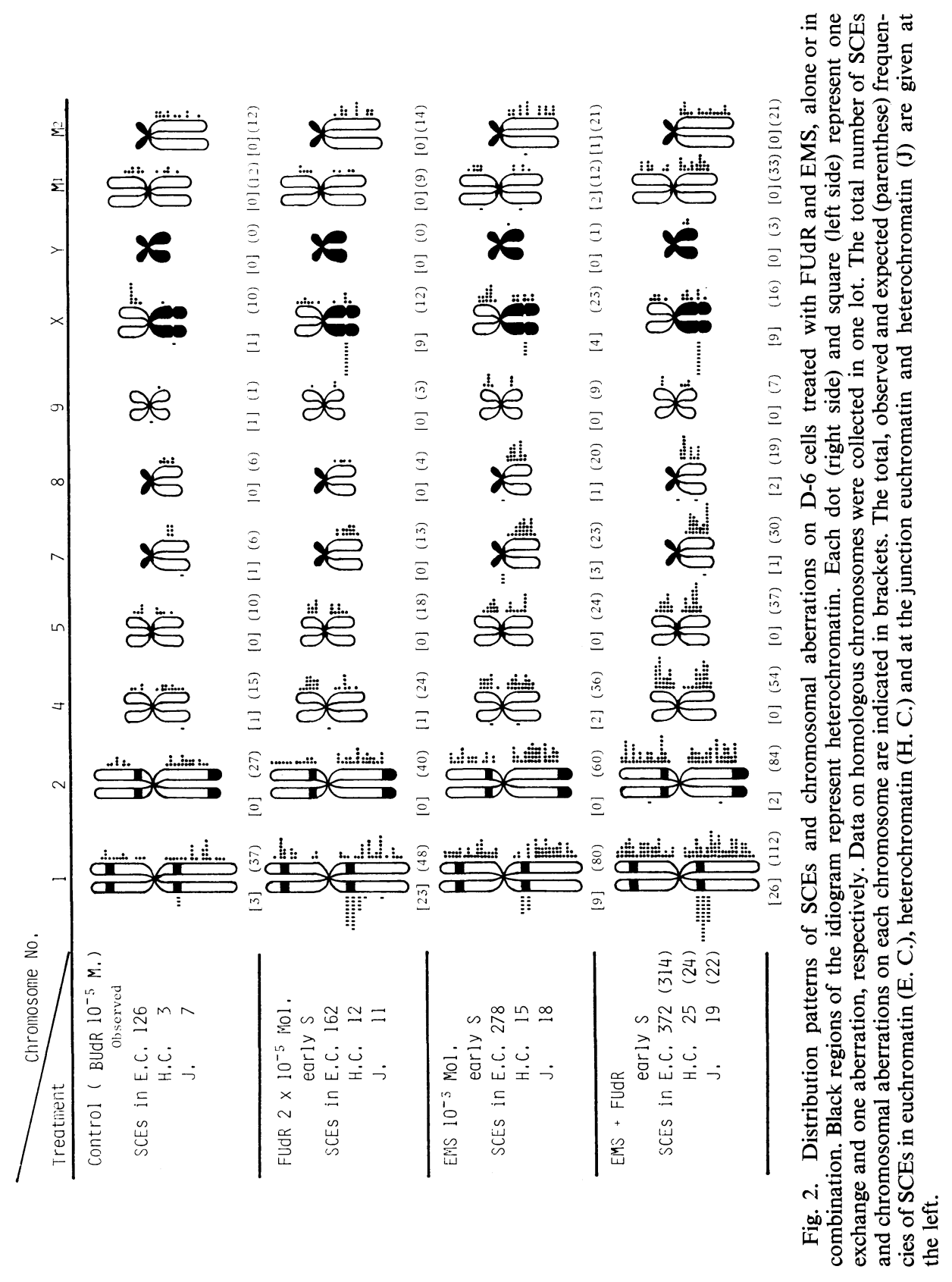


cy; the combined effect appeared to be synergistic.

The effects of FUdR and EMS, alone or in combination, differed considerably depending on the stage of cells, i.e., the early, middle or late $S$ phase, at which cells were affected. The early $\mathrm{S}$ phase was the most sensitive to both FUdR and EMS ;Table 2).

EMS, FUdR, or both in combination, had little or no effect on the SCE frequency in the heterochromatic region of the long arm of chromosome 1; also, combined treatment did not seem to enhance the SCE frequency in the heterochromatin of sex chromosomes because the values were somewhat reduced. By contrast, the chromosomal aberrations induced by these agents seemed to be concentrated on the heterochromatin, in the long arms of chromosome 1 and the $\mathrm{X}$ chromosome. No chromosomal aberration was seen in the $\mathrm{Y}$ chromosome (Table 2, Fig. 2).

Another general feature that emerged was that the distribution of FUdR- and EMSinduced SCEs varied along the chromosomes, with relatively more SCEs in the euchromatic and much fewer in the heterochromatic regions. The frequency in the euchromatic region appeared to be nearly proportional to the chromosomal length, and enhancement of the SCE frequencies that resulted from the combined effect seems to occur equally in the entire euchromatic region.

\section{DISCUSSION}

Our present results indicate that the mechanisms involved in the production of chromosomal aberrations are not the same as those involved in the production of SCEs, since no close correlation was observed between the frequency distributions of SCEs and chromosomal aberrations estimated for different chromosomal regions at different phases of DNA replication.

EMS has been suggested to cause damage in the pre- and post-replicational DNA regions, and many of these lesions are long-lived $(8,20)$. This may be due to the fact that the early S-phase was the stage most sensitive to the chemically induced SCEs, as shown in our results. The increased rate of SCEs after FUdR treatment in the present study may be explained by the hypothesis that SCE frequency depends to some extent on the BUdR concentration $(4,9,11,19)$, and that FUdR inhibits thymidine synthesis which, thus producing efficient incorporation of BUdR into chromosomes $(4,5)$.

That treatment with EMS and FUdR in the early S phase scarcely induces SCEs in the heterochromatic regions may reflect the fact that heterochromatin remains condensed throughout the cell cycle except for a brief replication period (17); since heterochromatin DNA is replicated at late $\mathrm{S}$, it may be less accessible to the damage which produces SCEs.

There are several reports that spontaneous SCEs occur more frequently at the junction between the $\mathrm{C}$-band positive and $\mathrm{C}$-band negative chromosome regions $(1,2,6$, $10,16,21)$. This seems to be partially applicable to chemically induced SCEs (3). However, in our present study, we found no such tendency; EMS-induced SCEs were distributed uniformly on the euchromatic regions. A similar relationship has been reported between the mitomycin-induced SCE frequency and DNA content in euchromatin (3).

Although combined treatment with FUdR and EMS synergistically enhanced SCE frequency, the distribution of SCEs and chromosomal aberrations rarely varied. No 
synergisms occurred in the case of chromosomal aberrations; the reason for this is unknown.

\section{REFERENCES}

1. Bostock, C. J. and S. Christie. Analysis of the frequency of sister chromatid exchanges in different regions of chromosomes of the kangaroo rat (Dipodomys ordii). Chromosoma (Berl.) 56, 275-287, 1976

2. Carrano, A. V. and S. Wolff. Distribution of sister chromatid exchanges in the euchromatin and heterochromatin of the Indian muntjac. Chromosoma (Berl.) 53, 361-369, 1975

3. Carrano, A. V. and G. R. Johnston. The distribution of mitomycin C-induced sister chromatid exchanges in the euchromatin and heterochromatin of the Indian muntiac. Chromosoma (Berl.) 64, 97-107, 1977

4. Davidson, R. L., E. R. Kaufman, C. E. Dougherty, A. M. Oufllette, C. M. Difolco and S. A. LATT. Induction of sister chromatid exchanges by BUdR is largely independent of the BUdR content of DNA. Nature 284, 74-76, 1980

5. Galloway, S. M. Ataxia telangiectasia: The effects of chemical mutagens and X-rays on sister chromatid exchanges in blood lymphocytes. Mutant. Res. 45, 343-349, 1977

6. Gibson, D. A. and D. M. Prescott. Frequency and sites of sister chromatid exchanges in rat kangaroo chromosomes. Exp. Cell Res. 86, 209-214, 1974

7. Kato, H. and T. H. Yoshida. Differential responses of several aneusomic cell clones to ultraviolet irradiation. Exp. Cell Res. 74, 15-20, 1972

8. KATO, H. Induction of sister chromatid exchanges by chemical mutagens and its possible relevance to DNA repair. Exp. Cell Res. 85, 239-247, 1974

9. Като, H. Spontaneous sister chromatid exchanges detected by a BUdR-labelling method. Nature 251, 70-72, 1974

10. Kato, H. Spontaneous and induced sister chromatid exchanges as revealed by the BUdRlabeling method. Int. Rev. Cytol. 49, 55-97, 1977

11. Mazrimas, J. A. and D. G. Stetska. Direct evidence for the role of incorporated BUdR in the induction of sister chromatid exchanges. Exp. Cell Res. 117, 23-30, 1978

12. Shiraishi, Y. and A. A. SandBerg. The relationship between sister chromatid exchanges and chromosome aberrations in Bloom's syndrome. Cytogenet. Cell Genet. 18, 13-23, 1977

13. Sono, A., K. Matsumoto, K. Hayano and K. Sakaguchi. Sister chromatid exchanges and chromosome aberrations induced by chemical agents in L5178Y cells. Cell Struct. Funct. 3, 249-254, 1978

14. Sono, A. and K. SAKaguchi. Induction of sister chromatid exchanges in Chinese hamster cells by various antitumor agents and its relation to the chromosome aberrations. Cell Struct. Funct. 3, 341-347, 1978

15. Sono, A. and K. Sakaguchi. Effects of inhibitors of nucleic acid and protein synthesis on ethyl methanesulfonate-induced sister chromatid exchanges in Chinese hamster cells. Cell Struct. Funct. 4, 75--80, 1979

16. Sparvoli, E. and H. Gay. Distribution of sister chromatid exchanges along the chromosomes of Haplopappus gracilis. Caryologia 26, 521-530, 1973

17. SperLing, K. and E. N. RaO. Mammalian cell fusion. V. Replication behaviour of heterochromatin as observed by premature chromosome condensation. Chromosoma (Berl.) 45, 121131, 1974

18. Sumner, A. T. A simple technique for demonstrating centrometric heterochromatin. Exp. Cell Res. 75, 304-306, 1972

19. Wolff, S. and P. Perry. Differential Giemsa staining of sister chromatids and the study of sister chromatid exchanges without autoradiography. Chromosoma (Berl.) 48, 341-353, 1974

20. WolfF, S., J. Bodycote and R. B. Painter. Sister chromatid exchanges induced in Chinese hamster cells by UV irradiation of different stages of the cell cycle: The necessity for cells to pass 
through S. Mutat. Res. 25, 73-81, 1974

21. Wolff, S. Sister chromatid exchanges. Ann. Rev. Genet. 11, 183-201, 1977

(Received for publication, May 16, 1980) 